\title{
Application of the Measured Equation of INVARIANCE TO RADIATION AND SCATTERING BY FLAT SURFACES
}

\author{
Rafael Pous; Mark Prouty, and Kennetl: K. Mei \\ EECS Dept., Univ. of California, Berkeley. CA 94720 \\ (R. Pous is now with the TSC Dept., Univ. Polit. de Catalunya, \\ Apdo. 30.002, 08080 Barcelona, Spain).
}

\begin{abstract}
The recently introduced concept of the Measured Eq tation of Invariance (MEI) $[1,2,3]$ is used to solve the problem of radiation and scattering by flat surfaces. Because on flat surfaces the electric currents are confined to two dimensions, a simple vector potential formulation can be used. The problem of radiation and scattering by rectangular strip dipoles is solved. including the transversal variation of the current across the dipole width. Also of interest are the currents induced on antemnas with step variations in width, and with bends and T-junctions.
\end{abstract}

The MEI is an equation that can be used to terminate a Finite Difference (FD) mesh arbitrarily close to the object of interest, avoiding the use of absorbing boundary conditions, which require the mesh to extend beyond the region of interest, with an increase in computation time and storage memory.

The mesh used to solve a strip dipole is a rectangular mesh which extends only a few cells (typicaliy 2 to 4 ) in each direction. The points of this mesh fall into three categories: exterior points or points on the mesh boundary, points on the surface of the dipole. and interior points not on the surface of the dipole. For each category a different type of FD equation will be used. The unknown is chosen to be the vector potential $\vec{A}$ defined as $\vec{H}^{s c a t}=\vec{\nabla} \times \vec{A}$ ( $\mu$ factored in for simplicity). If the flat surface is parallel to the $x y$ plane, only $A_{x}$ and $A_{y}$ need to be considered, and their values at each mesh point are the miknowns of the FD problem. Since two unknowns are associated with ach mesh point, two equations need to be written also.

For the interior points not on the metal surface, the standard FD approximation of the wave equation is used for each of the two potentials

$$
\left(\nabla^{2}+k_{o}^{2}\right) \cdot \mathcal{A}_{1}=0 \quad l=x, y
$$

"This research was sponwored by the California State MICRO program, and the industrial Sponsor Hughes Aircraft Company

$0-7803-1246-5 / 93 / \$ 3.00 @ 1993$ IEEE. 
For the points on the metal surface, the condition that $\vec{E}^{\text {sct }}=-\vec{E}^{\text {inc }}$ is used, yielding the equations

$$
\begin{aligned}
& \left(\frac{\partial^{2}}{\partial x^{2}}+\frac{\partial^{2}}{\partial y^{2}}+k_{o}^{2}\right) A_{x}=\frac{-j k_{o}}{Z_{o}} E_{z}^{i n c}+\frac{\partial H_{z}^{i n c}}{\partial y} \\
& \left(\frac{\partial^{2}}{\partial x^{2}}+\frac{\partial^{2}}{\partial y^{2}}+k_{o}^{2}\right) A_{y}=\frac{-j k_{o}}{Z_{o}} E_{y}^{i n c}-\frac{\partial H_{z}^{i n c}}{\partial x}
\end{aligned}
$$

This equations call easily be put in FD form. Special care needs to be taken at the edge of the flat surface. For the component of the vector potential that is parallel to the edge, a slight variation of the above equations needs to be used, and for the normal component, we use the fact that the normal current vanishes at the edge (see [4] for more details).

Finally, for the points at the mesh boundaries, we write an equation of the form

$$
A_{d 0}+\sum_{j=1}^{N-1} c_{j} \cdot A_{d j}=0 \quad d=x, y
$$

where $A_{d 0}$ is the value of the vector potential at the boundary point and $A_{d j}$ are the values at the $N-1$ neigliboring points ( $N$ may be 4,5 , or 6 depending on whether the point is on a side, an edge, or a corner of the mesh $)$. The coefficients $c_{i}$ are calculated to make Eq11. 4 a least sguare error fit for the $M$ potentials (which we call measuring functions)

$$
A_{d}^{i}(\bar{r})=\int_{S} J_{d}^{t}\left(\bar{r}^{\prime}\right) \cdot \frac{e^{-j k_{n}\left|\vec{r}-\bar{r}^{\prime}\right|}}{4 \pi\left|\bar{r}-\bar{r}^{\prime}\right|} \cdot d S^{\prime} \quad d=x, y \quad i=1 \ldots M=M_{x} \times M_{y} \geq N-1(5)
$$

where $J_{x}^{i}, J_{y}^{i}$ are two sets of linearly independent currents, which we call metrons. For a rectangular plate of length $l$ and width $w$, these metrons are

$$
\begin{aligned}
& J_{x}^{m_{x} m_{y}}(x, y)=\sin \frac{m_{x} \pi x}{l} \cdot \cos \frac{\left(m_{y}-1\right) \pi y}{w} \quad 1 \leq m_{x} \leq M_{x} \quad 1 \leq m_{y} \leq M_{y} \quad(6) \\
& J_{y}^{m_{x} m_{y}}(x, y)=\cos \frac{\left(m_{x}-1\right) \pi x}{l} \cdot \sin \frac{m_{y} \pi y}{w} \quad 1 \leq m_{x} \leq M_{x} \quad 1 \leq m_{y} \leq M_{y} \quad(7)
\end{aligned}
$$

Figure 1 shows the longitudinal current on a strip dipole of length and width $l=18.5 w=1.42 \lambda$, when fed off-center at $l / 4$, and the input admittance of a centerfed thin strip dipole. Figure 2 shows the currents in the two directions when the twisted dipole is fed at the center. Both the longitudinal and transversal variations of the currents are obtained, as well as a clear picture of how the currents behave near the 90 degree bend. Figure 3 shows the monostatic radar cross section of a square plate of side $a$, compared to the measurements reported in [6].

\section{References}

[1] K. K. Mei, R. Pous, Z. Chen, Y. W. Liu, and M. D. Prouty, "The Measured Equation of Invariance: a new concept in field computation," IEEE Trans. Antennas Propagat., submitted for publication. 

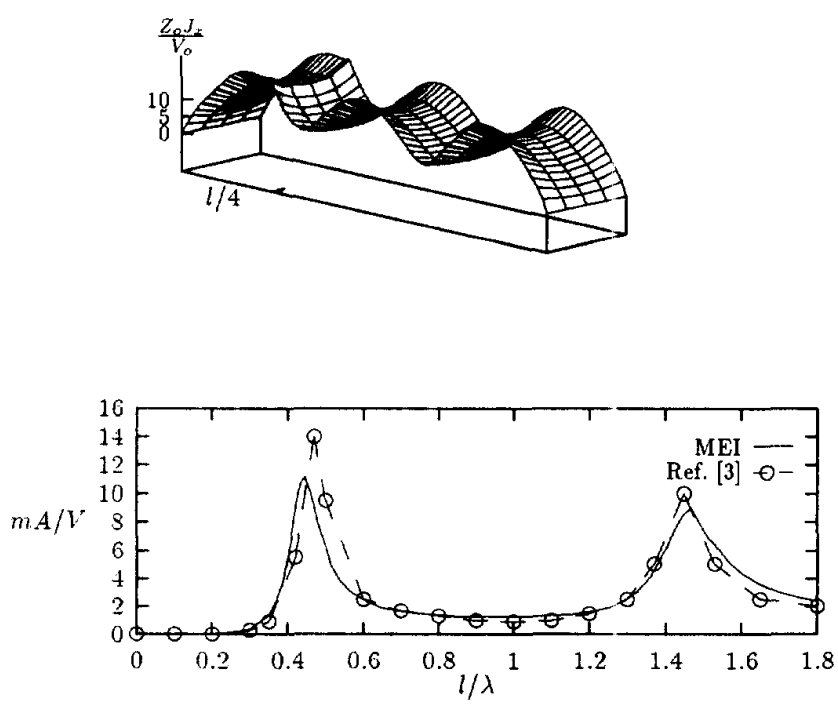

Figure 1: Current on an off-center fed strip dipole antenna with $l=18.5 w=1.42 \lambda$, fed at $l / 4$ (top) and input admittance of a center-fed strip dipole antenna with $l=$ $18.5 w$ versus frequency, compared with the MOM result presented in [5] (bottom).

[2] R. Pous, "The Measured Equation of Invariance: a new concept in field computation," Ph.D. dissertation, Univ. of California at Berkeley, 1992.

[3] K. K. Mei, R. Pous, M. D. Prouty, and Y. W. Liu, "Further insight into the Measured Equation of Invariance," IEEE Antennas and Propagat. Intl. Sumposium, Ann Arbor, Michigan, 1993.

[4] M. D. Prouty, R. Pous, and I. K. Mei, "Application of the Measured Equation of Invariance to transmission lines and discontinuities," IEEE Antennas and Propagat. Intl. Symposium, Ann Arbor, Micligan, 1993

[5] R. F. Harrington, Field Compntation by Moment Metiıods, Robert E. Krieger Publishing Company. Malabar, Florida, 1982.

[6] S. M. Rao, D. R. Wilton, and A. W. Glisson, "Electromagnetic scattering by surfaces of arbitrary shape," IEEE Trans. Antennas and Propagat., vol. 30, pp. 409-418, May 1982. 

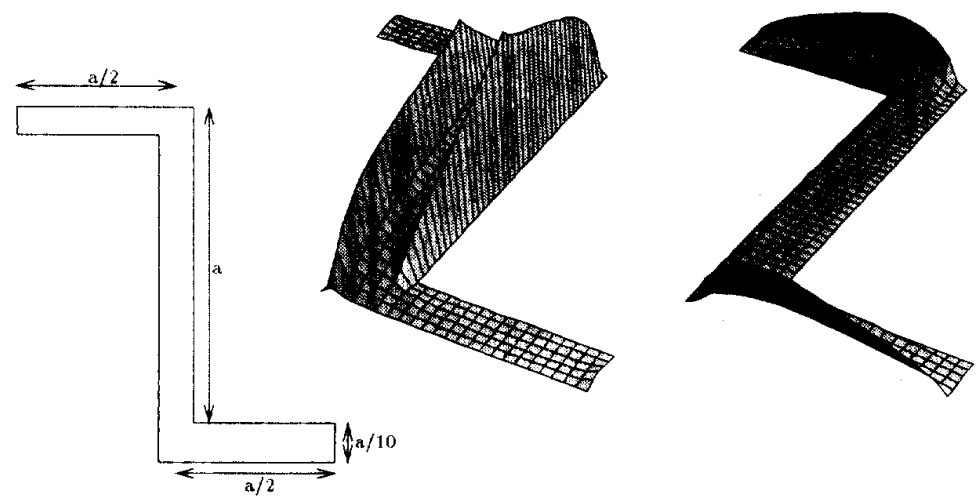

Figure 2: Currents on a twisted dipole, for $\lambda=4 a$ (vertical urrent on the left, and horizontal current on the right) .

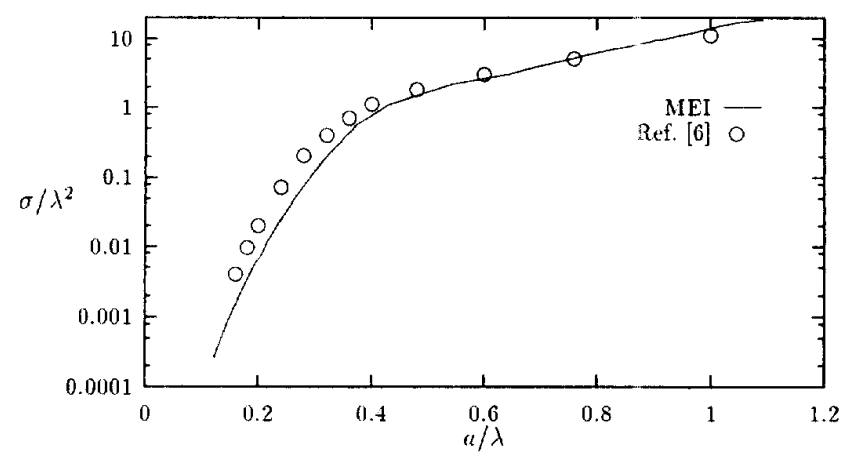

Figure 3: Monostatic malas cross section vs. frequency for at square plate of side $a$, for normal incidence. compared to the measurements reported in [6] (the calculation is for a zero-thickness plate, and the measurements are for a plate of thickness $0.000127 \lambda)$. 